\title{
Extended Transsphenoidal
}

\section{Approach with $\Sigma$-shape Osteotomy of the Maxilla: Technical Note}

\begin{abstract}
The traditional sublabial transsphenoidal approach offers a limited operative field. This report describes a simple surgical technique to widen the sublabial transsphenoidal window to access the midline lesions from the planum sphenoidale to the lower clivus. This technique was developed on the basis of clinical experience and on data obtained by cadaveric dissection study. Following a sublabial incision and separation of the bilateral septal mucosa, a small $\Sigma$-shape osteotomy of the maxilla, including the anterior nasal spine, was performed with a surgical saw. The width of the entrance to the nasal cavity ranged from 32 to $38 \mathrm{~mm}$. Although the plexus of the anterior superior alveolar nerve was partially cut, our clinical experience revealed no significant sensory loss of the incisors after the $\Sigma$-shape osteotomy. The nasal crests of the maxilla and palatine bone were drilled out, and the roof of the nasopharynx was then incised to expose the lower clival bone. Although extradural sellar or clival lesions were the focus of this approach, the intradural anatomical structures examined by cadaveric study were presented. The extended transsphenoidal approach with $\Sigma$-shape osteotomy of the maxilla is a simple technique and provides widened access to the sellar and clival regions.
\end{abstract}

The traditional sublabial transnasal transsphenoidal approach ${ }^{1,2}$ is a common and extremely efficient method for gaining access to the sellar and clival lesions. The disadvantages of the standard transsphenoidal approach include limited lateral and inferior exposure, and a deep and narrow field. ${ }^{2-4}$ This approach is therefore intrinsically incapable of treating certain parasellar or lower clival regions. Over the last decade, various midline or anterior cranial base approaches have been developed to access these regions, and some of the new methods have been utilized as an alternative to the transsphenoidal approach. The approaches that have been put to use include the transbasal, 5 transmaxillary, ${ }^{6-8}$ transsphenoethmoid, ${ }^{9}$ transoral, ${ }^{10,11}$ extended frontal, ${ }^{12}$ mandible-splitting transoral approaches, ${ }^{3}$ facial translocation, ${ }^{13}$ extended maxillotomy, ${ }^{14}$ and Le Fort I osteotomy. ${ }^{15,16}$ These radical procedures provide wide exposure with better illumination and improved vision, but involve complex techniques, extensive dissection, and the potential for significant morbidity. 4,17

Some experienced surgeons have continued to advocate the use of the transsphenoidal approach in the treatment of midline cranial base tumors such as clival chordoma. ${ }^{3,4}$ Among the over 2000 cases in the senior author's (T.F.) personal experience, in which the transsphenoidal procedures were applied, approximately 100 cases required various modifications of the procedure, including several types of maxillotomies. To

Skull Base Surgery, Volume 9, Number 2 Department of Neurosurgery, Allegheny University of the Health Sciences, Center for Skull Base Surgery, Pittsburgh, PA (Y.S., S.T., T.F.), and Department of Neurosurgery, Hokkaido University Graduate School of Medicine, Sapporo, Japan (Y.S.). Reprint requests: Dr. Sawamura, Department of Neurosurgery, Hokkaido University School of Medicine, North-15, West-7, Kita-ku, Sapporo 060-8638, Japan. Copyright $\odot 1999$ by Thieme Medical Publishers, Inc., 333 Seventh Avenue, New York, NY 10001, USA. Tel.: +1 (212) 760-0888 x 132. 1052-1453/1999/E 1098-9072(1999)09:02:0119-0126:SBS 00143X 
simply expand the very narrow and deep window obtained by the standard transsphenoidal procedure, we have investigated an appropriate and minimally invasive modification of the standard approach, and have concluded that a $\Sigma$-shape osteotomy of the maxilla has a great advantage in this regard. The present report describes a surgical technique for exposing the midline region from the planum sphenoidale to the lower clivus through sublabial-transseptal-transsphenoidal route. A cadaveric prosection study was carried out to reevaluate clinical experience and to confirm anatomical structures exposed by this technique.

\section{MATERIALS}

Seven adult cadaveric specimens fixed in Notox, which is composed of an aqueous solution containing ethanol (TLV 1000 ppm) bis carbonyl compounds, propylene glycerol, and antiseptic and antifungal agents, were examined in this study. The carotid and vertebral arteries were injected with red silicon, and the internal jugular veins were injected with blue silicon. Each specimen was placed in a three-pins holder system to approximate as closely as possible the positioning of a real patient in an actual surgical set up. Dissections were performed grossly and under $\times 4$ to $\times 40$ magnification with a surgical microscope. All observations were made from the surgeon's angle of view, and procedures were consistent with what would be done in a clinical setting.

\section{SURGICAL TECHNIQUE}

This technique has been established by clinical and cadaveric studies. The patient position during this procedure and the stages of the surgical procedure are similar to those of the standard transsphenoidal approach. A horizontal sublabial incision is made above the mucogingival reflection from the first premolar on either side. The periosteum is elevated to expose the anterior nasal spine, septal cartilage, and the lower circumference of the pyriform aperture, including inferomedial part of the maxillary buttress, but not the infraorbital foramen (Fig. 1A). The nasal mucosa is detached from the septum at the midline and the floor, while the inferior turbinates are left intact. The nasal cartilage is disarticulated from the vomer.

Using an oscillating saw with a thin blade, the horizontal incision of the maxilla is performed first (Fig. 1B). A pantorthogram of the dentition is advisable to identify the height of the dental roots and so that they can be avoided when the incision is performed. The horizontal incision line is made at 4 to $5 \mathrm{~mm}$, usually $5 \mathrm{~mm}$, downward from the inferior edge of the pyriform aperture, as shown in Figures 1B and 1C. The vertical incision is carried out at the lateral edge of the pyriform aperture. The medial walls of the maxillary sinus are not violated. The neural plexus of the anterior superior alveolar nerves supplying to the incisors is partially preserved. These incisions result in removal of the $\Sigma$-shape bone piece of the maxilla (Fig. 1C). The width of the bone piece ranged from 32 to $38 \mathrm{~mm}$.

The sphenoid sinus is exposed just as in the standard transseptal transsphenoidal approach. The mucosa of the nasal floor is detached along the nasal crests of the maxilla and palatine bone toward the dorsal end of the palatine bone, which is the anterior edge of the roof of the epipharynx. The crests of the maxilla and palatine bone are totally drilled out to facilitate horizontal access to the roof of the epipharynx, and subsequently to the middle clivus (Fig. 1D). To access to the lower clival bone, the roof and posterior wall of the pharynx is incised at the midline. The pharyngobasilar fascia is detached from the clival bone by use of cutting of monopolar diathermy.

Whether one should extend the bone removal around the sellar and clivus depends on the character of the specific lesion to be treated. With the removal of the anterior bony wall of the sella, the sellar tubercle, the limbus sphenoidale, and the posterior part of the planum sphenoidale, the extended transsphenoidal approach provides a wide window around the pituitary gland with better illumination (Fig. 2A). The bony prominences of the optic nerve and the cavernous carotid artery are easily identified. Exposure of the anteroinferior medial venous space of the cavernous sinus and the anterior bend of the internal carotid artery is possible, but carries a risk of bleeding (Fig. 2B), and was performed only when a tumor invaded and filled the venous space.

The amount of clival bone removal depends on the extent of tumor involvement. It is possible to gain access to the lower clivus, although the caudal edge of the clivus, the foramen magnum, cannot be manipulated in direct vision. The middle clival bone can be removed, exposing the dura mater, approximately 15 to $20 \mathrm{~mm}$ in width (Fig. 3A). Lateral access is limited by the pterygoid bone and the internal carotid artery. The abducens nerve, which runs in the venous space of the Dorello's canal (Fig. 3B), may be observed at the lateral limit of the removed clival bone before the dural opening, as indicated by the arrow in Figure 3C. Clival tumors often manifest abducens palsy at this location, and therefore special care should be taken not to injure the nerve when performing tumor removal.

Although this approach aims at extradural sellar or clival lesions, Figure 3C presents a panoramic view of the intradural anatomical structures after dural opening. At the end of the procedure, the incised roof of the epipharynx is sutured. The $\Sigma$-shaped bone piece includ- 
A
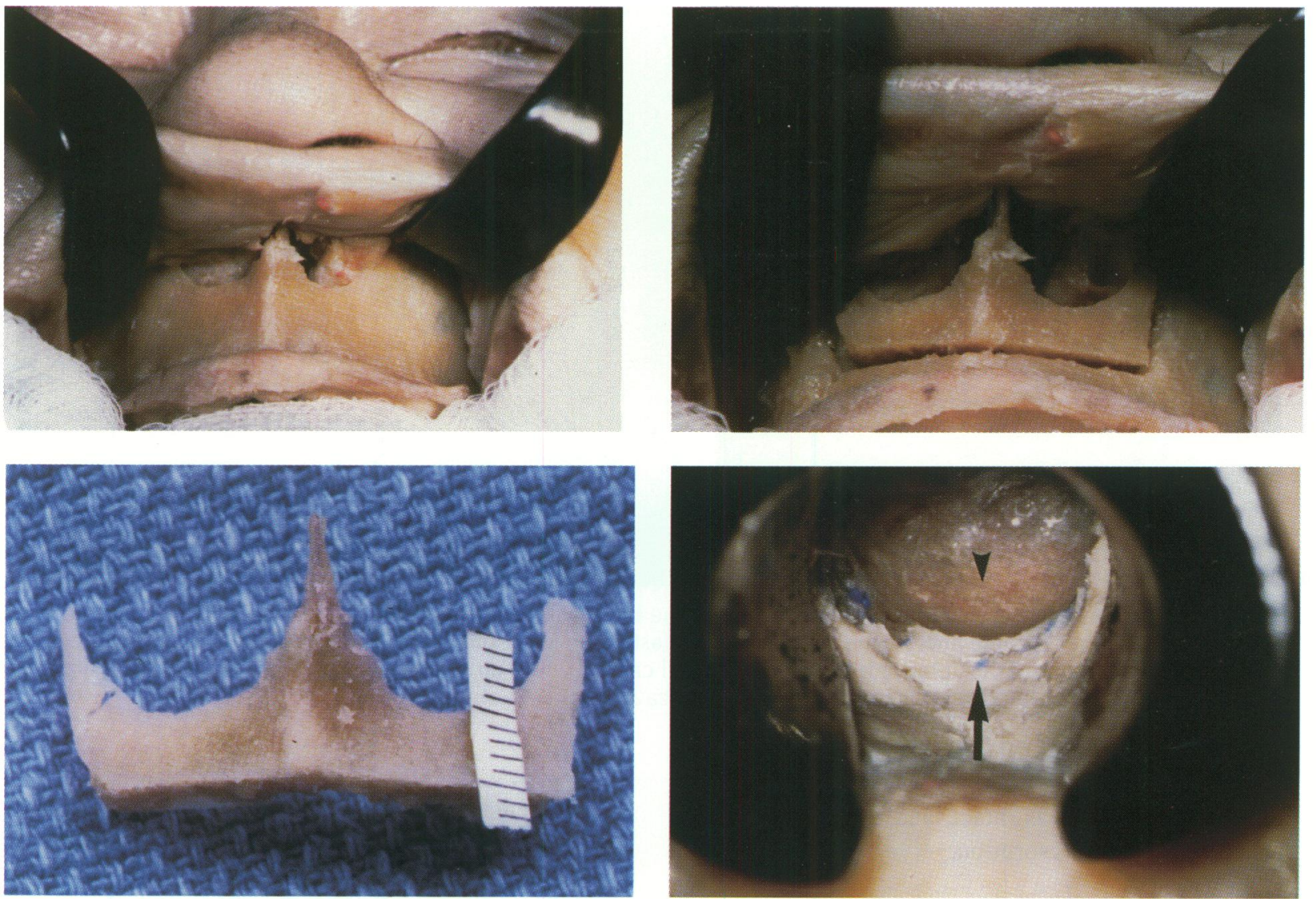

Figure 1. A horizontal sublabial incision was made above the mucogingival reflection from the first premolar on either side, and the periosteum was then elevated to expose the anterior nasal spine and the lower circumference of the pyriform aperture (A). Using an oscillating saw with a thin blade, the horizontal incision of the maxilla was performed first avoiding injury to the dental roots, and then the vertical incision was made, preserving the medial wall of the maxillary sinus (B). A bone piece in $\Sigma$-shape was removed (C). After removal of the anterior wall of the sphenoid sinus, the middle clivus (arrow head) and the roof of the epipharynx (arrow) appeared (D).

ing the nasal spine is replaced and fixed in position over the maxillary buttress with titanium miniplates.

\section{ILLUSTRATIVE CASE}

A 55-year-old man presented with a 3-month history of double vision. On admission, a neurological examination revealed solely left abducens nerve palsy. Magnetic resonance imaging (MRI) and computerized tomography (CT) scanning depicted a mass involving the entire clival bone and extending to the sphenoid sinus, but not to the foramen magnum (Figs. 4A and 4D). With a preoperative diagnosis of a clival chordoma, the patient underwent tumor removal by the extended transsphenoidal approach.

The sphenoid bone ventral to the tumor was removed by rongeur and a high-speed drill, for which there was an enough working space. The mass was ex- tremely hard, much like a tendon, and was firmly adherent to bone tissue inside the expanded clival bone. This firm tumor could not be debulked by curette, and was resected using rongeur, microscissors with alligatortype shanks, and a drill. The dorsal cortex of the clival bone was mostly intact, and the dura mater anterior to the brain stem was not exposed. The abducens nerve was not observed; that probably situated behind connective tissue network. This tumor was almost completely resected, with only a lateral part of the mass left on the right side.

The patient was able to have oral intake on the day following surgery. The postoperative clinical course was uneventful, and the left abducens nerve palsy completely disappeared within a week. Because the histologic diagnosis was a desmoid tumor, we adapted a wait-and-see policy based on its nature. Two months after surgery, a follow-up MRI showed a normal configuration of the epipharyngeal space (Fig. 4C). The sensa- 
A
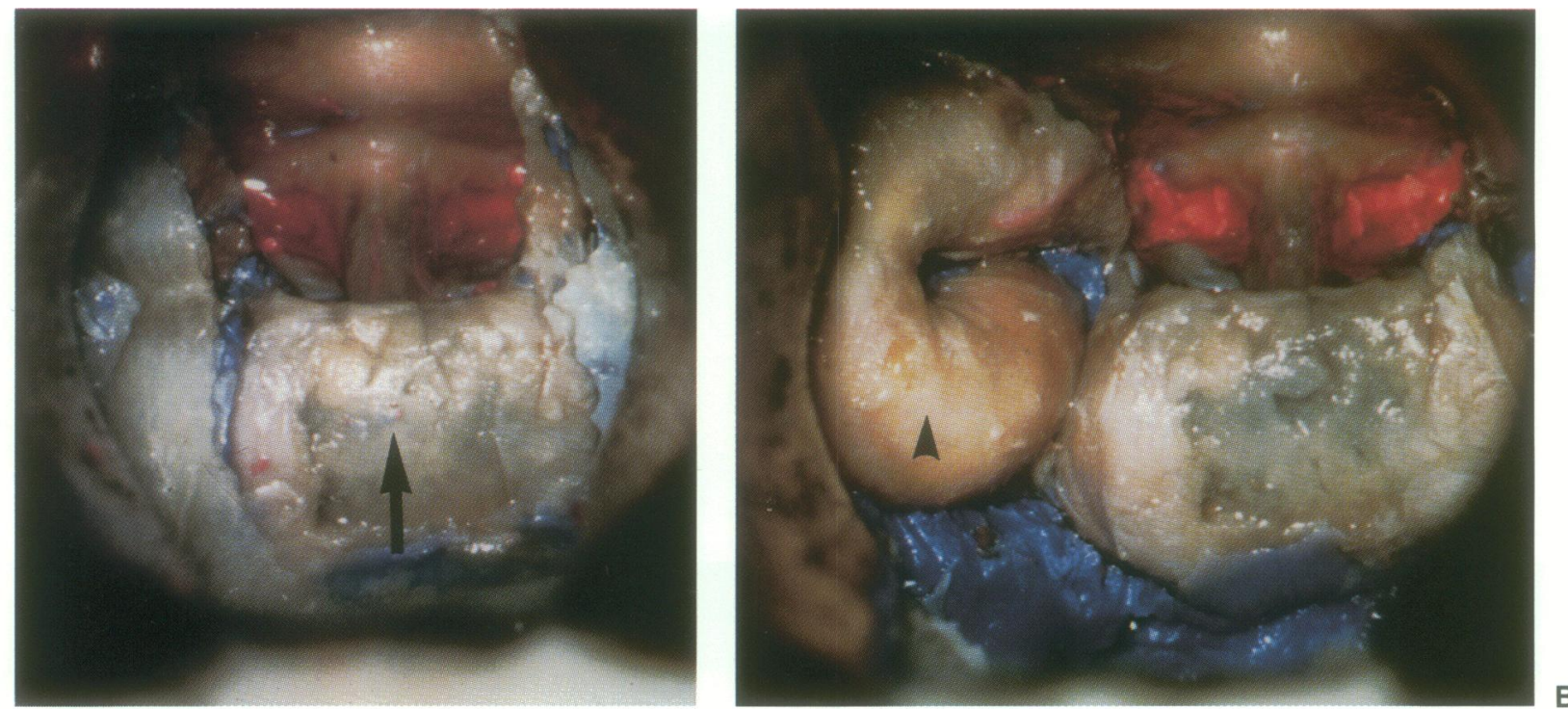

Figure 2. The sella turcica and the tuberculum sellae have been removed (A). The extended transsphenoidal approach provides a better view around the pituitary gland (asterisk). Exposure of the anteroinferior medial venous space of the cavernous sinus and the anterior bend of the internal carotid artery (arrow head) is possible (B); however, it carries a risk of bleeding. The blue dye indicates the inferior intercavernous sinus.

tion of the incisors was intact, and the $\Sigma$-shaped bone piece fused without resorption.

\section{DISCUSSION}

The choice of surgical approach in treating sellar and clival lesions depends on many factors, including tumor location, clinical status of the patient, previous surgery and/or radiation, and the experience and philosophy of the surgeon. ${ }^{4}$ Radical anterior cranial-base approaches can offer better access to a given lesion, but there is a high chance of surgical morbidity. Possible morbidity includes malocclusion, necrosis of the anterior maxilla, necrosis of the alveolar bone, loss of tooth vitality, subcutaneous emphysema, spontaneous pneumomediastinum, perforation or deviation of the nasal septum, abducens nerve palsy, and hemorrhage. ${ }^{818-22}$ In addition, the loss of position sense in the upper teeth by denervation of the anterior superior alveolar nerves predisposes the patient to malocclusion.

The selection of an optimal approach for a given tumor is not absolute, but is rather a relative decision. The standard transsphenoidal approach is the prevailing method for providing exposure to the sella turcica and the upper and middle clivus with no significant surgical morbidity caused by the approach itself.1,2 Our technique will further increase the utility of the standard approach.

A small $\Sigma$-shape osteotomy around the pyriform aperture is a simple and less invasive technique in terms of maximum preservation of the skeletal framework and neurovascular structures. This partial maxillotomy leaves the maxillary and ethmoid sinuses intact, and fracturing the maxilla is not necessary. Malocclusion or lack of vascular supply to the maxilla and palatine bone do not occur. The $\Sigma$-shape osteotomy adds some advantages to the standard transsphenoidal procedure, such as a wider window than that provided by the standard procedure. Observation around the sella turcica is remarkably improved with the better illumination. The improved procedure also can enable us to access the lower clivus, excluding the foramen magnum. Through the wide corridor, drilling of the sphenoid bone became an easier procedure.

Although, our technique itself has the possibility of devitalizing the upper front teeth and of causing secondary nasal deformity because of the resection of the nasal spine. However, a careful procedure will allow us to avoid these complications. A pantorthogram of the dentition is advisable to identify the height of the dental roots and so that they can be avoided when the bone incision is performed. Because the horizontal incision line is made at 4 to $5 \mathrm{~mm}$ downward from the inferior edge of the pyriform aperture, a part of the anterior superior alveolar nerves, which actually make a plexus in the maxilla bone, can be preserved. We would like to emphasize that our technique should not be utilized in a routine fashion because of the possible risk for devitalization of the incisors. However, for the type of case discussed in this report, this technique has significant advantages over the much more extensive transmaxillary and facial degloving-type approaches. Although, the simple approach that removes the amount of bone with rongeurs or with drilling has been used and pre- 
A
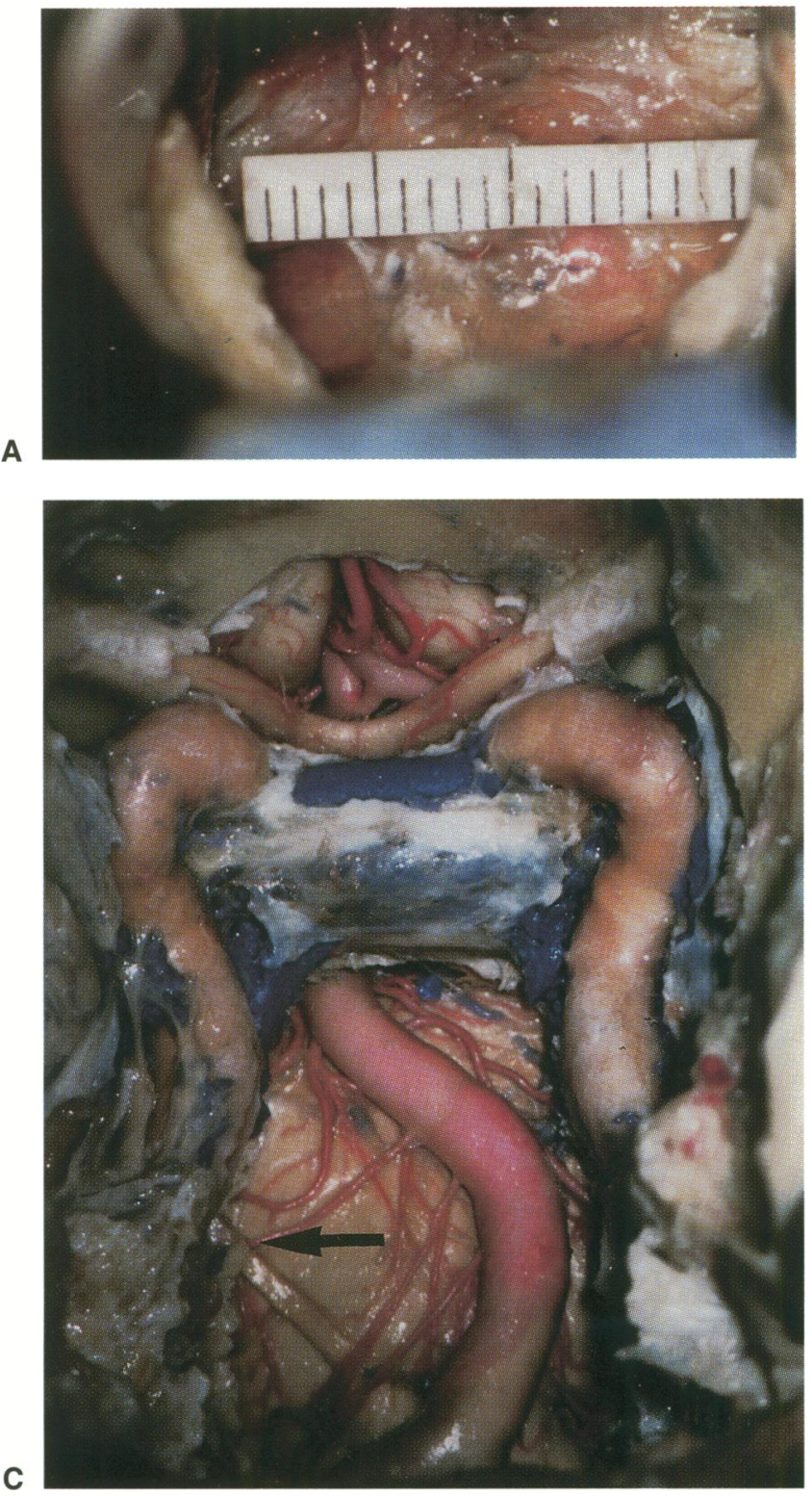

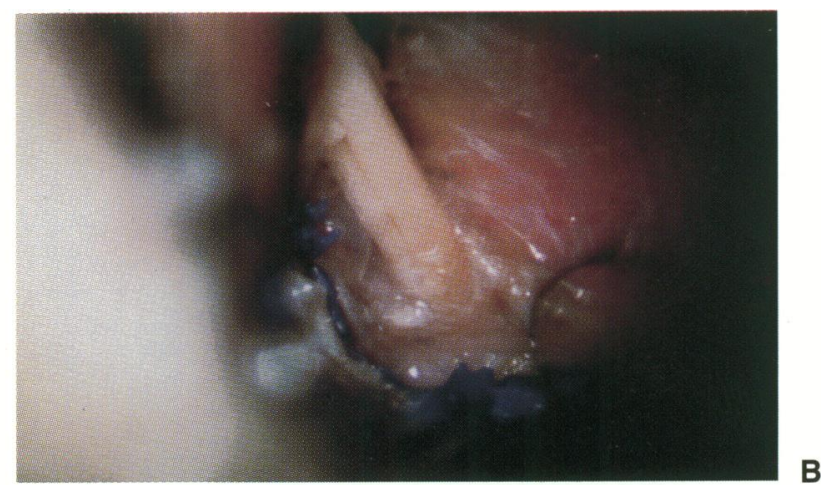

Figure 3. The middle clival bone has been removed exposing the dura mater, approximately 15 to $20 \mathrm{~mm}$ in width (A). Before dural opening, the abducens nerve runs in venous space of the Dorello's canal (B). Although this approach aims at extradural sellar or clival lesions, the intradural anatomical structures are presented in a panoramic view $(\mathbf{C})$ : arrow indicates the abducens nerve at the entrance of the Dorello's canal.

be resected, at least in part, by sharp or flexible curettes, but a removal of harder tumor as in the illustrative case may be difficult. The extended transsphenoidal approach allowed us to remove this tendinous-firm desmoid tumor. Neuroendoscopic observation through the wide window will further assist in the removal of clival tumors.

\section{CONCLUSION}

The extended transsphenoidal approach with the $\Sigma$-shape osteotomy of the maxilla is a simple technique and provides wider exposure of the sellar and clival regions. This technique does not increase the risk of morbidity of the standard transsphenoidal approach. 

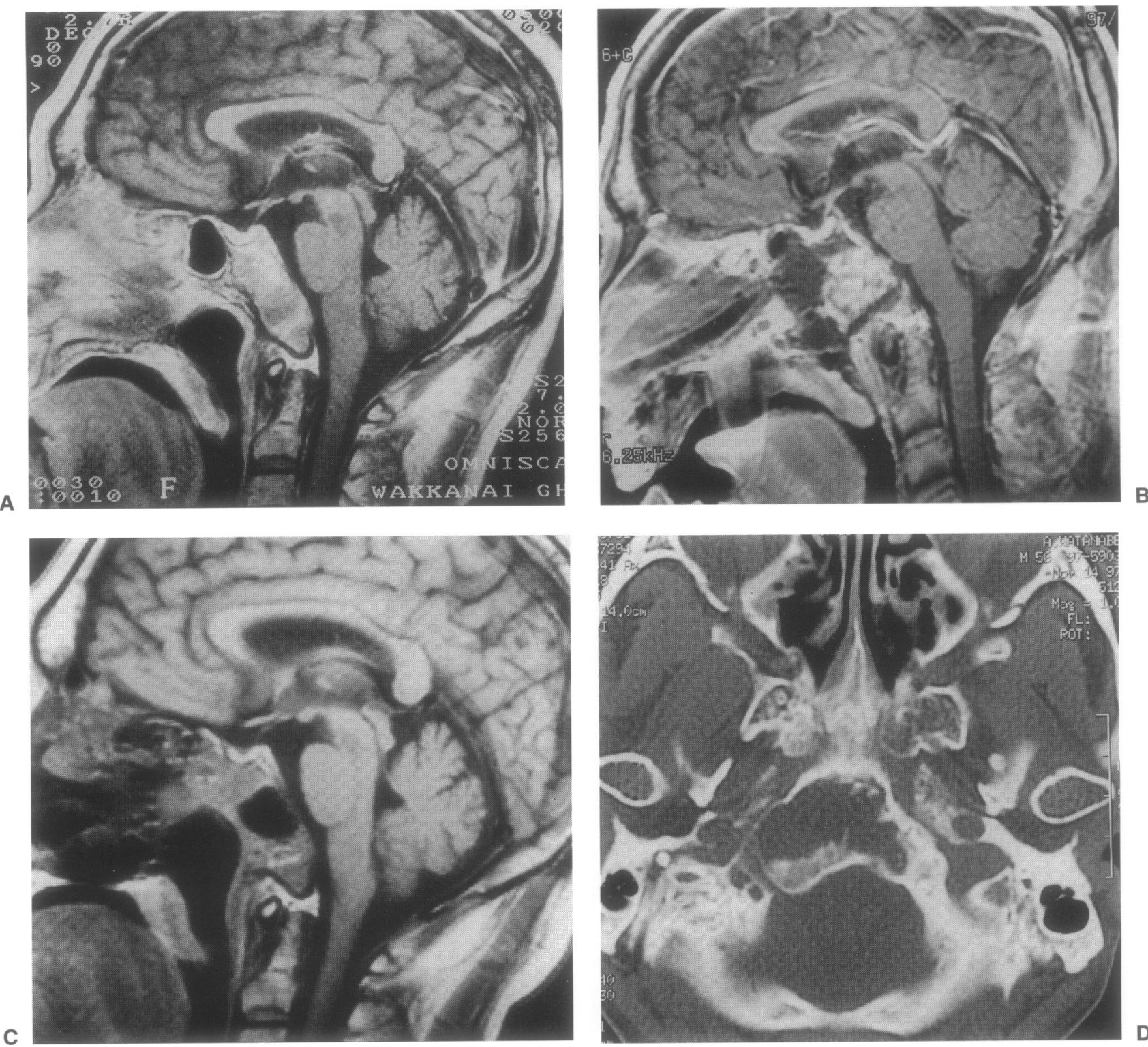

D

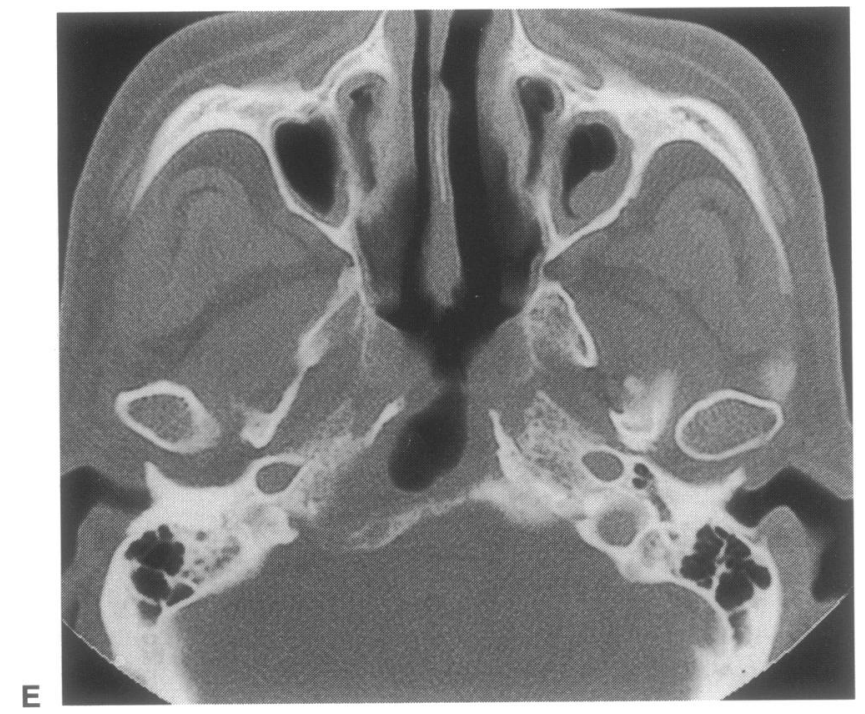

Figure 4. Sagittal MRI showing a clival desmoid tumor involving the entire clivus and causing left abducens palsy (A). MRI taken 1 day after surgery demonstrates a surgical intervention just above the anterior arch of the atlas (B). Two months after surgery, the epipharyngeal space retrieved its normal configuration (C). Axial CT images show the extent of bone removal at the conjunction of the middle and lower clivus [(D) preoperative $C T$ and (E) postoperative $C T$ ]. This tendinous firm tumor was almost completely resected leaving a lateral part of the mass on the right side. 


\section{ACKNOWLEDGMENT}

The authors thank Dr. Shouren Shih for excellent preparation of cadaveric specimens.

\section{REFERENCES}

1. Hardy J. Transsphenoidal microsurgery of the normal and pathological pituitary. Clin Neurosurg 1969;16:185-217

2. Landolt AM, Strebel P. Technique of transsphenoidal operation for pituitary adenomas. In: Krayenbühl $\mathrm{H}$, ed. Advances and Technical Standards in Neurosurgery, Vol. 7. Vienna: Springer-Verlag, 1980, 119-177

3. Laws ER Jr. Clivus chordomas. In: Sekhar LN, Janecka IP, eds. Surgery of Cranial Base Tumors. New York: Raven Press, 1993, 679-685

4. Maira G, Pallini R, Anile C, et al. Surgical treatment of clival chordomas: The transsphenoidal approach revisited. J Neurosurg 1996;85:784-792

5. Derome PJ. The transbasal approach to tumors invading the skull base. In: Schmidek HH, Sweet WH, eds. Operative Neurosurgical Techniques, Vol. 1. New York: Grune \& Stratton, 1988, 619-633

6. Couldwell WT, Sabit I, Weiss MH, Giannotta SL, Rice D. Transmaxillary approach to the anterior cavernous sinus: A microanatomic study. Neurosurgery 1997;40:1307-1311

7. Fraioli B, Esposito V, Santoro A, et al. Transmaxillosphenoidal approach to tumors invading the medial compartment of the cavernous sinus. J Neurosurg 1995;82:63-69

8. Rabadán A, Conesa H. Transmaxillary-transnasal approach to the anterior clivus. A microsurgical anatomical model. Neurosurgery 1992;30:473-482

9. Lalwani AK, Kaplan MJ, Gutin PH. The transsphenoethmoid approach to the sphenoid sinus and clivus. Neurosurgery 1992 ; $31: 1008-1014$
10. Crockard HA, Sen CN. The transoral approach for the management of intradural lesions at the craniovertebral junctions: Review of 7 cases. Neurosurgery 1991;28:88-98

11. Delgado TE, Garrido E, Harwick RD. Labiomandibular transoral approach to chordomas in the clivus and upper cervical spine. Neurosurgery 1981;8:675-679

12. Sekhar LN, Nanda A, Sen CN, Synderman CN, Janecka IP. The extended frontal approach to tumors of the anterior, middle, and posterior skull base. J Neurosurg 1992;76:198-206

13. Janecka IP, Nuss DW, Sen CN. Facial translocation approach to the cranial base. Acta Neurochir (Wien) 1991;53(Suppl):193198

14. James D, Crockard HA. Surgical access to the base of skull and upper cervical spine by extended maxillotomy. Neurosurgery 1991;29:411-416

15. Sasaki CT, Lowlicht RA, Astrachan DI, et al. Le fort I osteotomy approach to the skull base. Laryngoscope 1990;100:10731076

16. Uttley D, Moore A, Archer DJ. Surgical management of midline skull-base tumors: A new approach. J Neurosurg 1989;71: 705-710

17. Gay E, Sekhar LN, Rubinstein E, et al. Chordomas and chondrosarcomas of the cranial base: Results and follow-up of 60 patients. Neurosurgery 1995;36:887-897

18. Lanigan DT, West RA. Management of postoperative hemorrhage following the Le Fort I maxillary osteotomy. J Oral Maxillofac Surg 1984;42:367-375

19. Parner EI, Becker ML. Necrosis of the anterior maxilla following osteotomy. Oral Surg 1972;33:326-330

20. Stringer DE, Dolwick MF, Steed DL. Subcutaneous emphysema after Le Fort I osteotomy. report of two cases. J Oral Surg 1979;37:115-116

21. Watts PG. Unilateral abducens nerve palsy: A rare complication following a Le Fort I maxillary osteotomy. Br J Oral Maxillofac Surg 1984;22:212-215

22. Westwood RM, Tilson HB. Complications associated with maxillary osteotomies. J Oral Surg 1975;33:104-115 